Journal of

Molecular Microbiology

and Biotechnology
J Mol Microbiol Biotechnol 2017;27:29-42

DOI: $10.1159 / 000450725$
Received: May 10. 2016

Accepted: September 9, 2016

Published online: January 21, 2017

\title{
Requirements for Septal Localization and Chromosome Segregation Activity of the DNA Translocase SftA from Bacillus subtilis
}

\author{
Nina El Najjar ${ }^{a}$ Christine Kaimer ${ }^{b}$ Thomas Rösch ${ }^{a} \quad$ Peter L. Graumann ${ }^{a}$ \\ a SYNMIKRO, LOEWE Center for Synthetic Microbiology, and Department of Chemistry, Philipps-Universität Marburg, \\ Marburg, and ${ }^{b}$ Fakultät für Biologie und Biotechnologie, Lehrstuhl Biologie der Mikroorganismen, Ruhr University \\ Bochum, Bochum, Germany
}

\section{Keywords}

Bacterial cell cycle $\cdot$ Chromosome segregation · DNA translocase $\cdot$ Bacillus subtilis

\begin{abstract}
Bacillus subtilis possesses 2 DNA translocases that affect late stages of chromosome segregation: $\mathrm{SftA}$ separates nonsegregated DNA prior to septum closure, while SpollIE rescues septum-entrapped DNA. We provide evidence that $\mathrm{Sft} A$ is associated with the division machinery via a stretch of 47 amino acids within its $\mathrm{N}$-terminus, suggesting that $\mathrm{Sft} A$ is recruited by protein-protein interactions with a component of the division machinery. SftA was also recruited to mid-cell in the absence of its first 20 amino acids, which are proposed to contain a membrane-binding motif. Cell fractionation experiments showed that SftA can be found in the cytosolic fraction, and to a minor degree in the membrane fraction, showing that it is a soluble protein in vivo. The expression of truncated SftA constructs led to a dominant sftA deletion phenotype, even at very low induction rates of the truncated proteins, indicating that the incorporation of nonfunctional monomers into SftA hexamers abolishes functionality. Mobility shift experiments and surface plasmon binding studies showed that SftA binds to DNA in a cooperative manner, and demonstrated low ATPase activity when binding to short nucleotides rather than to long stretches of DNA.

(c) 2017 S. Karger AG, Basel
\end{abstract}

(c) 2017 S. Karger AG, Basel

\section{Introduction}

Bacterial cell division occurs with remarkable fidelity across a wide range of environmental conditions. However, certain types of stress can damage DNA and disrupt the normal progression of the replication fork. Therefore, cells have evolved pathways that coordinate the process of cell division with DNA replication. This ensures that cell division occurs reliably even when segregation is perturbed [Crozat et al., 2014; Kaimer and Graumann, 2011]. DNA translocation is also important for the resolution of chromosome dimers, which occur in $15 \%$ of the cells due to uneven recombination events between sister chromosomes [Crozat et al., 2014], and which lead to a block in the cell cycle.

In Escherichia coli, the membrane-integral ATP-dependent translocase FtsK is recruited as a regular component of the division septum via its $\mathrm{N}$-terminal domain, which is essential for cell division. The protein is anchored in the membrane via its central membrane spans, and its C-terminal domain mediates DNA translocation. FtsK functions in segregating sister chromosomes during the late stages of the cell cycle in a wide range of bacteria [Barre, 2007; Bigot el al., 2007; Stouf et al., 2013] through activating site-specific recombination by XerCD at dif [Aussel et al., 2002]. The motor domain FtsK $_{C}$ is composed of $\alpha-, \beta$-, and $\gamma$-subdomains. The $\alpha$ - and

\section{KARGER}

E-Mail karger@karger.com

www.karger.com $/ \mathrm{mmb}$
Peter L. Graumann

SYNMIKRO, LOEWE Center for Synthetic Microbiology, and Department of Chemistry Philipps-Universität Marburg

Hans-Meerwein-Strasse 6, DE-35032 Marburg (Germany)

E-Mail peter.graumann@ @ynmikro.uni-marburg.de 
$\beta$-subdomains form a double-stranded DNA (dsDNA) translocase [Massey et al., 2006]. The $\gamma$-subdomain plays a role in the recognition of the FtsK orientating polar sequence (KOPS) that guides FtsK toward the dif site at ter [Sivanathan et al., 2006], as well as in the activation of XerCD-dif recombination [Aussel et al., 2002]. KOPS are distributed over the chromosome and oriented toward the terminus region, where they are found at a high frequency [Bigot et al., 2005; Bobay et al., 2013]. FtsK arranges the duplicated dif sites in close proximity at the division septum facilitating dimer resolution [Bigot et al., 2005].

Activation of recombination requires direct interaction between the $\gamma$-subdomain and XerD [Yates et al., 2006]. XerC and XerD bind the dif site located within the terminus region, ter, unlink catenated chromosomes, and resolve chromosome dimers formed by homologous recombination [Barret and Sherratt, 2005; Grainge et al., 2007; Hallet et al., 1997; Sherratt et al., 1995].

Single-molecule experiments using DNA curtains revealed that $\mathrm{FtsK}_{\mathrm{C}}$ can push, evict, and bypass proteins bound to DNA as it translocates. $\mathrm{FtsK}_{\mathrm{C}}$ stops at least transiently and/or dissociates at XerCD bound to dif [Collins et al., 2014; Graham et al., 2010; Lee et al., 2014]. FtsK locates KOPS through random collisions, preferentially in the ADP-bound state, and is incapable of recognizing KOPS while translocating along DNA [Lee et al., 2012]. Reversals in translocation direction were previously reported to occur spontaneously and in response to XerCD bound to dif [Lee et al., 2012; Pease et al., 2005]. However, in a recent study employing singly tethered labeled DNA as well as labeled $\mathrm{FtsK}_{\mathrm{C}}, \mathrm{FtsK}_{\mathrm{C}}$ translocation and its interaction with synaptic complexes of XerCD on the single molecule level was observed. $\mathrm{FtsK}_{\mathrm{C}}$ seemed to assemble on DNA as a single hexamer, which then translocated rapidly. Upon reaching XerCD-bound dif, whether in a synapsed or unsynapsed conformation, it resided briefly and then dissociated without reversal. FtsK $\mathrm{K}_{\mathrm{C}}$ activated recombination when it met synapsed XerCD-dif complexes, and then dissociated before the completion of recombination by XerCD [May et al., 2015].

In Bacillus subtilis, RipX and CodV are the homologues of XerD and XerC, respectively, and share 35 and $44 \%$ sequence identity with their E. coli counterparts [Sciochetti et al., 1999]. Both RipX and CodV were reported to bind to the B. subtilis dif site and to catalyze strand exchange in vitro [Sciochetti et al., 2001].

Two B. subtilis DNA translocases share homology with FtsK and are involved in the rescue of DNA that might become trapped by the division septum during vegetative growth: the membrane-associated SpoIIIE protein and the soluble SftA protein. The 2 proteins participate synergistically in dimer resolution through coordinating chromosome segregation and cell division at different stages of the cell cycle in B. subtilis [Kaimer et al., 2011]. $\mathrm{Sft} \mathrm{A}$ is a component of the divisome. It translocates DNA during septation, while SpoIIIE is exclusively recruited to rescue septum-entrapped DNA after division is completed [Biller and Burkholder, 2009; Kaimer et al., 2009; Sharpe and Errington, 1995].

Localization in different genetic backgrounds indicated that SftA is probably recruited as an early divisome component [Biller and Burkholder, 2009; Kaimer et al., 2009]. Biochemical characterization showed that the fulllength protein forms hexamers, but the isolated C-terminal translocase domain was monomeric, which means it is the $\mathrm{N}$-terminus that is capable of favorizing hexamerization [Kaimer et al., 2009]. In E. coli, it was shown that 50 residues overlapping the $\mathrm{N}$-terminus and the linker region were involved in the stabilization of an active hexameric form of FtsK [Aussel et al., 2002]. Although the C-terminal domain of SftA (residues 490-952) shares 50 and $56 \%$ identity to DNA translocases FtsK and SpoIIIE respectively, including the ATP and DNA binding motifs, the glutamate- and proline-rich N-terminus (residues 1-439) shows no homology to any of these translocases [Kaimer et al., 2009; Kaimer and Graumann, 2011]. While no transmembrane-spanning regions were predicted, a recent review suggested the existence of 3 putative $\mathrm{N}$-terminal transmembrane helices based on BlastP analysis of 2,385 sequences of FtsK homologues thought to lack a transmembrane domain [Crozat et al., 2014]. SftA midcell localization depends on the initiation of cell division by FtsZ and is most likely based on direct protein-protein interaction via its $\mathrm{N}$-terminal domain.

In this work, we investigate the specific region of SftA required for septum recruitment, as well as the in vitro DNA binding and ATPase activity of the protein. We find that $\mathrm{Sft} A$ contains a region within its $\mathrm{N}$-terminus that is required for stable anchoring at the septum. We also show that SftA binds cooperatively to DNA, in agreement with its formation of DNA-binding hexamers.

\section{Results and Discussion}

\section{SftA Is a Soluble Protein}

$\mathrm{SftA}$ can be produced as a soluble enzyme in vitro [Kaimer et al., 2009]. To test if SftA is membrane-attached, or also soluble, in vivo, we performed cell fractionation experiments. Figure 1a shows that $\mathrm{Sft} A$ is predominantly 


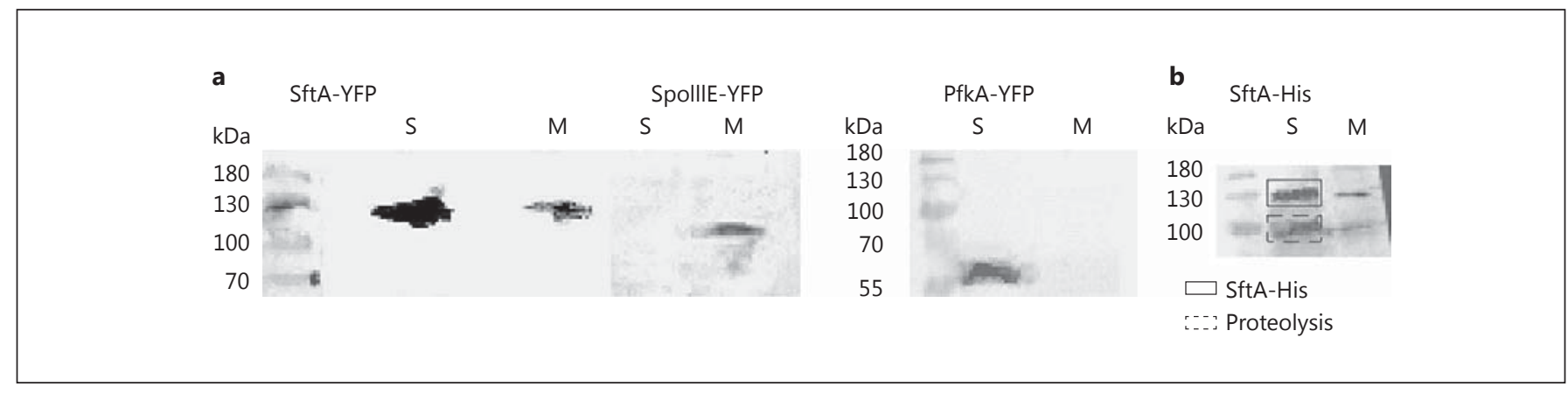

Fig. 1. a Western blot with anti-GFP antibodies for the detection of YFP-tagged proteins of SftA-YFP, SpoIIIE-YFP, and PfkA-YFP expressed from their original promoters in the soluble $(S)$ and membrane (M) fractions of each corresponding B. subtilis culture following cell fractionation experiments. $\mathbf{b}$ Western blot with anti-
His antibodies for the detection of overexpressed His-tagged SftA in the soluble (S) and membrane (M) fractions of an E. coli BL21 strain harboring SftA in a pETDUET-1 vector under an IPTGinducible promoter. present in the soluble fraction of B. subtilis exponential cells, with a minor fraction of the protein being associated to the membrane. As controls, SpoIIIE, a membrane-integral DNA translocase, and PfkA, a soluble glycolytic enzyme (phosphofructokinase), were used [Burton and Dubnau, 2010; Ludwig et al., 2001]. As expected, SpoIIIE was exclusively found in the membrane fraction, while PfkA was solely in the cytosol (Fig. 1a). The same experiment was done in parallel on E. coli BL21 cells harboring a vector containing a His-tagged SftA. The protein was once again detected in the cytosolic fraction, as well as in the membrane fraction, albeit to a much lesser extent (Fig. 1b). These experiments show that SftA is largely soluble within the cell.

Forty-Seven Amino Acids of the N-Terminus of SftA

Can Localize the Protein to Mid-Cell in B. subtilis

In order to narrow down the region of the $\mathrm{N}$-terminus responsible for septal localization, the domain was truncated into fragments of decreasing length and the localization pattern of each construct was investigated in $B$. subtilis. The fragments were cloned into PSG1164y, were integrated into the B. subtilis chromosome and were expressed from the original gene locus. Thereby, truncated SftA variants are expressed under the control of the original promoter, while full-length SftA can be expressed by the xylose promoter that is inside pSG1164y. A map depicting the vector construction and integration is displayed in online supplementary Figure S1 (see www. karger.com/doi/10.1159/000450725 for all online suppl. material). All the truncations were chosen at random. The N-terminus of SftA [Sft $\mathrm{A}_{(1-439)}$-yellow fluorescent protein (YFP)] showed a normal septal localization pattern in B. subtilis when expressed from the amyE locus (Fig. 2b). Figure $2 \mathrm{a}$ shows a map of the various truncations included in this study, while Figure $2 b-i$ shows the localization of these truncations. The first 246 residues $\left.\left(\mathrm{SftA}_{(1-246)}\right)^{-} \mathrm{YFP}\right)$ showed a localization pattern similar to that of the wild-type protein (Fig. 2d). Fluorescence was detected in all cells. The truncation localized to the new septum in $50 \%$ and to the cell middle in $31 \%$ of the cells, mostly forming 2 adjacent dots within the membrane, while $19 \%$ of the cells $(n=400)$ showed faint lateral fluorescence (Fig. 2d). Of note, $4-5 \%$ of the cells expressing the truncation showed segregation defects, e.g. nonsegregated nucleoids in cells larger than $3.7 \mu \mathrm{m}$, which is never observed in wild-type cells, suggesting that the presence of an SftA truncation interferes with the function of wildtype SftA (see below).

$\mathrm{SftA}_{(1-137)}$-YFP also localized normally (Fig. 2e). The same was observed for a shorter truncation, whereby $\mathrm{SftA}_{(1-105)}-\mathrm{YFP}$ also localized to the septum in $48 \%$ of the cells, the cell middle in $26 \%$, and the rest showed lateral localization ( $\mathrm{n}=500$; Fig. 2f). $\mathrm{SftA}_{(1-67)}$-YFP also localized in around $60 \%$ of the cells $(n=372)$, mostly to the new septa and to the mid-cell (Fig. $2 \mathrm{~g}$ ).

It is possible that a fragment of the $\mathrm{N}$-terminus of SftA can localize through hexamerization with full-length SftA. The original copy of the gene was deleted in 2 different truncation-variant strains in order to investigate the role of complex formation with full-length protein. Truncations 105 and 67 were each cloned into pSG1193y and were integrated into the $a m y E$ locus via a double crossover event. The original sftA was deleted by trans- 


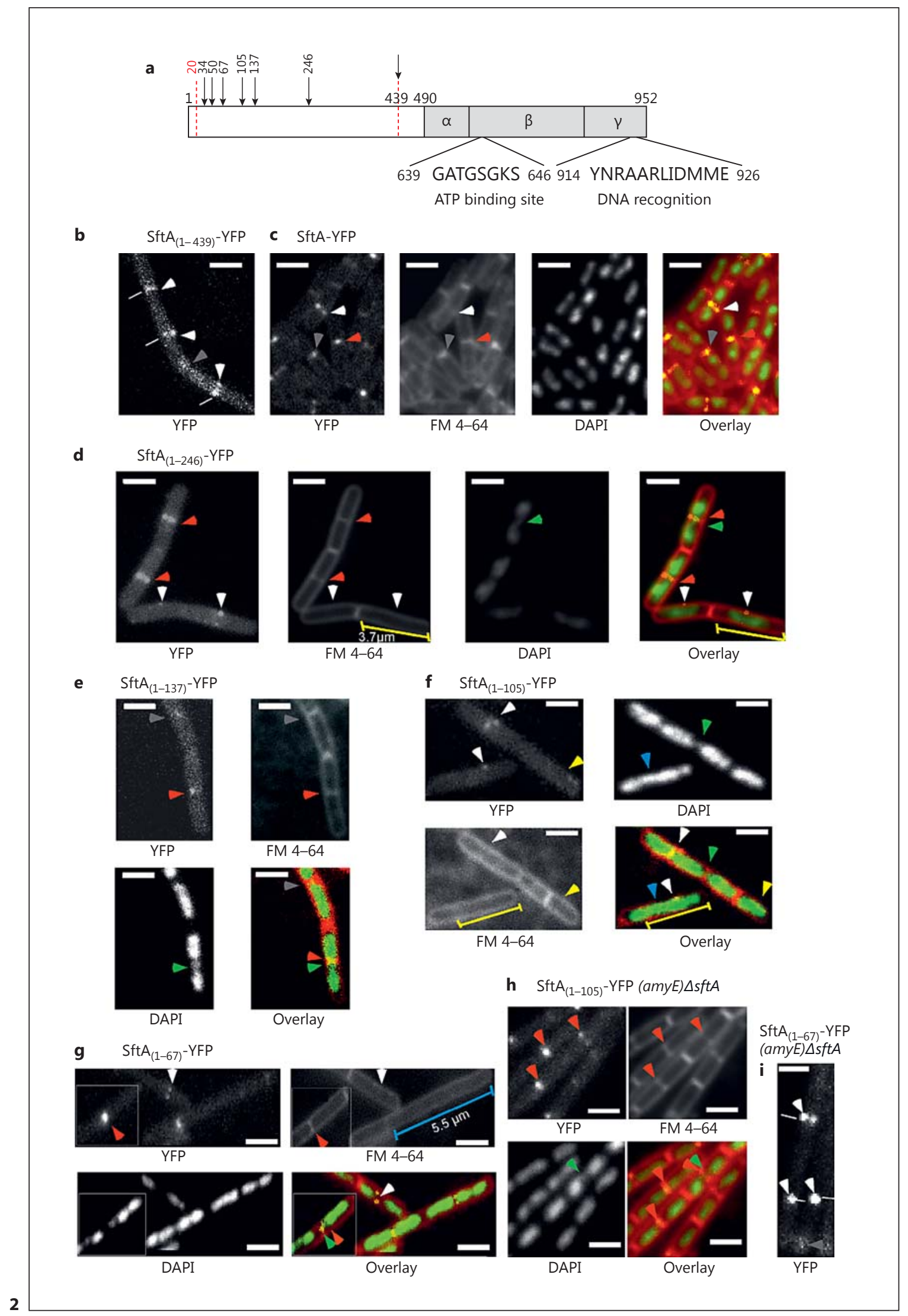

(For legend see next page.) 
forming the resulting $B$. subtilis truncation strains with genomic DNA from strain CK 150 (sftA::tet) [Kaimer et al., 2009]. The strains were grown to exponential phase in $\mathrm{S}_{50}$ fructose, induced with $0.05 \%$ xylose for $1 \mathrm{~h}$ and then analyzed. The 105-bp truncation [ $\mathrm{Sft}_{(1-105)}$-YFP] localized to the septa and mid-cell both before and after deletion of the original $\mathrm{sftA}$ at rates of $79 \%(\mathrm{n}=400)$ and $85 \%$ $(\mathrm{n}=300)$, respectively (Fig. $2 \mathrm{f}, \mathrm{h})$. Likewise, the 67-residue-long fragment [SftA $\mathrm{S}_{(1-67)}$-YFP] localized normally when expressed from the amyE locus in the presence of a full-length original copy of the gene, and foci were detected in $88 \%$ of the cells $(\mathrm{n}=318$; Fig. $2 \mathrm{~g})$. The deletion of the original sftA did not affect this localization pattern (Fig. 2i). This finding suggests that the first 67 residues of the SftA N-terminus are capable by themselves of an interaction with an unidentified partner that integrates the protein into the septum early in cell division. Deleting the foremost 20 residues of the $\mathrm{N}$-terminus of SftA also did not affect the localization (Fig. 3a). A truncation $\mathrm{SftA}_{(21-105)}$-YFP was capable of localizing normally, whether in a wild-type or an $s f t A$ deletion background (Fig. 3b, c). Sft $\mathrm{A}_{(21-67)}$-YFP localized normally in a wildtype background, but showed an aberrant localization in the deletion background, whereby foci were only seen in $50 \%$ of the cells (Fig. $3 \mathrm{~d}$, e) in contrast to $80 \%$ for fulllength SftA-YFP. This experiment shows that the stretch between 20-67 residues is sufficient for normal septal targeting, and suggests that hexamerization with wild-type SftA contributes to mid-cell recruitment. Deleting the first 60 obliterated septal recruitment (Fig. 3g), and the truncations shorter than 67 residues did not localize, as is seen in Figure $3 \mathrm{~h}$ and i, for $\mathrm{Sft}_{(1-50)^{-}}$YFP and $\mathrm{Sft}_{(1-34)^{-}}$
Fig. 3. Localization of $\operatorname{Sft}_{(21-439)}$-YFP (a), $\operatorname{SftA}_{(21-105)}-\mathrm{YFP}$, and $\mathrm{SftA}_{(21-67)}-\mathrm{YFP}$ (b-e) both in a wild-type and an $s f t A$ deletion background. g-i The nonlocalizing truncations: a fluorescence picture of the $\mathrm{Sft}_{(61-439)}$ YFP and a Western blot of the construct with anti-GFP antibodies (g); $\mathrm{SftA}_{(1-50)}$-YFP (h) and $\mathrm{SftA}_{(1-34)}$-YFP (i). As a comparison, $\mathbf{f}$ shows background fluorescence levels of wild-type (WT) B. subtilis, which appear to be lower than the fluorescence detected in nonlocalizing truncations. White dashes indicate the mid-cell, white arrowheads indicate mid-cell localization, while grey arrowheads point to old cell poles. Scale bars, $2 \mu \mathrm{m}$. a $\quad \mathrm{SftA}_{(21-439)}$-YFP (amyE)

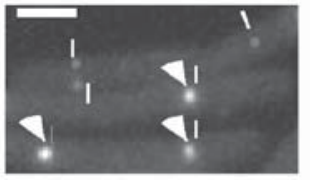

YFP

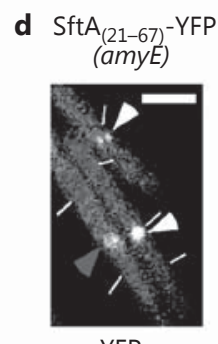

YFP

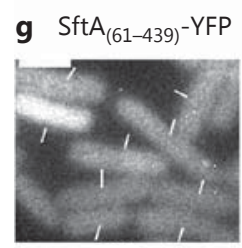

YFP

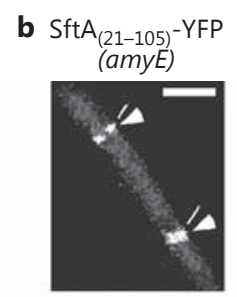

YFP

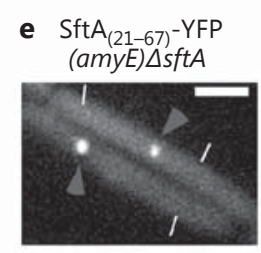

YFP c $\quad$ SftA $_{(21-105)^{-Y F P}}$

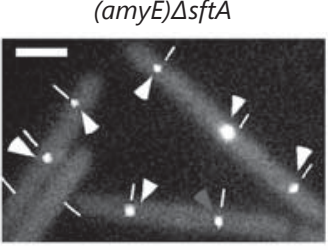

YFP

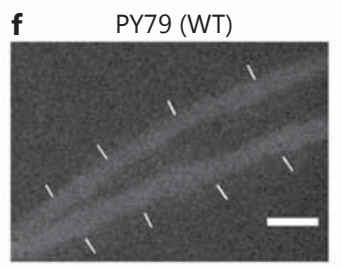

YFP

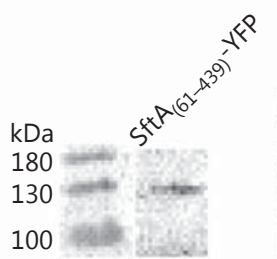

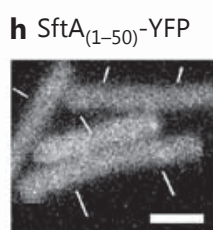

YFP

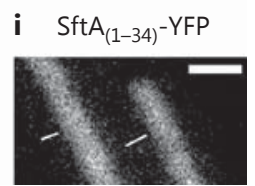

YFP
Fig. 2. a Map depicting the length of the different truncations. The red dashed lines represent the whole $\mathrm{N}$-terminus of the protein except for the first 20 residues. b-i Localization patterns of the truncations in a wild-type background. The yellow ruler represents $3.7 \mu \mathrm{m}$, and the blue ruler represents $5.5 \mu \mathrm{m}$. White arrowheads point to the mid-cell, red to new cell poles, grey to old poles, green to nucleoids bisected by a septum, and blue to unsegregated nucleoids in elongated cells. White dashes indicate the mid-cell in YFP images for which no FM 4-64 is shown. Scale bars, $2 \mu \mathrm{m}$.
Localization and Segregation of DNA

Translocase SftA from B. subtilis
J Mol Microbiol Biotechnol 2017;27:29-42 DOI: $10.1159 / 000450725$ 
Table 1. Phenotypes of wild-type, truncations, and mutant cells

\begin{tabular}{|c|c|c|c|c|c|c|}
\hline Strain & $\%$ cut $^{\mathrm{a}}$ & $\%$ long $^{\mathrm{b}}$ & $\%$ anucleate & $\%$ mononucleate $^{c}$ & $\%$ abnormal & Cells, $\mathrm{n}$ \\
\hline$w t$ & 0 & 0.7 & 0.1 & 2 & 1.1 & 1,025 \\
\hline$s f t A-y f p$ & 0.3 & 1.1 & 0 & 3 & 1.85 & 1,000 \\
\hline$s f t A_{(1-105)}-y f p$ & 1.16 & 1.97 & 0 & 27 & 7.18 & 860 \\
\hline $\begin{array}{l}s f t A_{(1-246)^{-}} y f p \\
a m y E:: s f t A_{(1-105)^{-}} y f p^{\mathrm{e}}\end{array}$ & 1.03 & 1.9 & 0 & 25 & 6.7 & 1,160 \\
\hline $0.02 \%$ & 0.97 & 1.43 & 0 & 26.6 & 6.39 & 977 \\
\hline $0.05 \%$ & 1.2 & 1.8 & 0 & 27.1 & 7.06 & 1,500 \\
\hline $0.5 \%$ & 1.22 & 2 & 0 & 29 & 7.57 & 1,800 \\
\hline sftA::tet & 1.4 & 2.13 & 0 & 26 & 7.6 & 1,500 \\
\hline
\end{tabular}

${ }^{\mathrm{a}}$ DNA bisected by a septum. ${ }^{\mathrm{b}}$ Cells longer than $5.5 \mu \mathrm{m} .{ }^{\mathrm{c}}$ In cells $3.7-5.5 \mu \mathrm{m}$ (i.e., $15 \%$ of wild-type cells, so the value is the percentage of $15 \%$ of all cells from the culture). ${ }^{\mathrm{d}}$ Cells induced with $0.05 \%$ xylose. ${ }^{\mathrm{e}}$ amyE::sftA $(1-105)-y f p$ with different xylose induction concentrations.

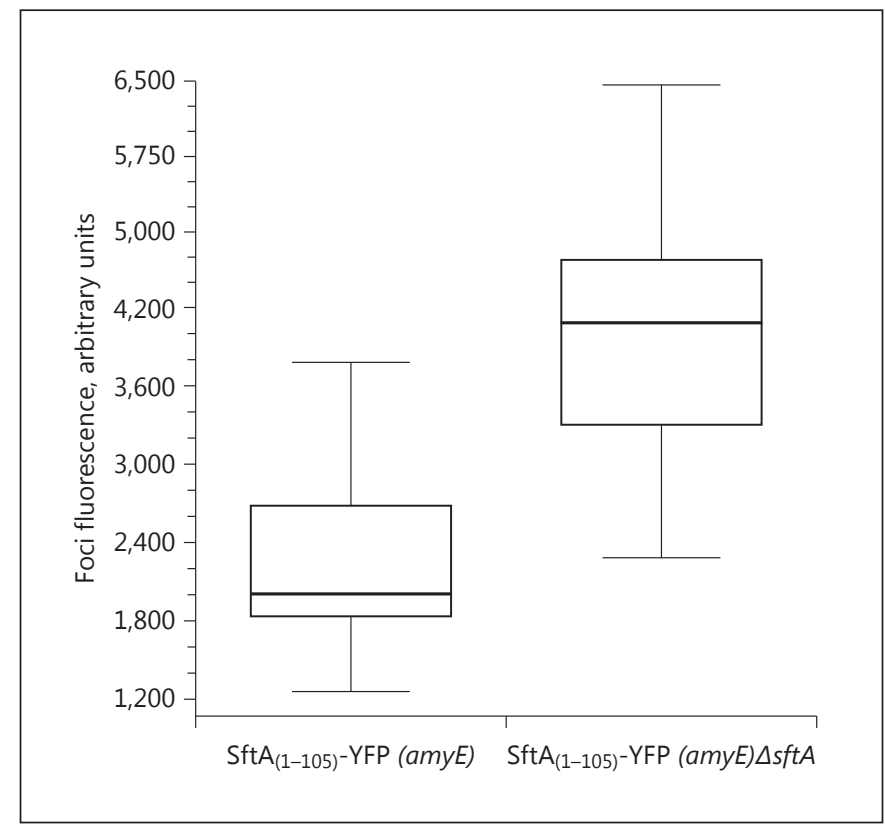

Fig. 4. Corrected fluorescence intensity for $\mathrm{SftA}_{(1-105)}$ YFP foci in the presence of the $s f t A$ original locus and after the deletion of the original locus. The boxes represent the values between the first and the third quartiles $(\Delta s f t A)$, the middle line being the median. The error bars show the lowest and highest values.

YFP, respectively. It has been shown for FtsK in E. coli that the protein lacking all transmembrane segments still allows the efficient resolution of chromosome dimers if it is connected to a septal targeting peptide through a sufficiently long linker. This means that a direct membrane insertion is not needed for functional translocation [Dubarry and Barre, 2010], but an efficient septal targeting is.

\section{Stoichiometry and Functionality}

For all the truncation constructs mentioned so far, the phenotypic outcome of their expression was similar to that of an sftA deletion, whether an original copy of the gene existed or not - all of the merodiploid strains had DNA trapped by a septum or showed nonsegregated nucleoids in cells larger than $3.7 \mu \mathrm{m}$ in about $5 \%$ of all cells, indicative of a segregation defect, and had a subset of elongated cells, all of which is typical of an sftA deletion (Fig. 2; Table 1). To confirm the idea that the incorporation of a truncated SftA monomer into a hexamer abolishes functionality, the corrected fluorescence of $\mathrm{SftA}_{(1-105)}$-YFP foci was calculated in the presence of an intact untagged SftA, expressed from the original locus, and was compared to the fluorescence of the same truncation after deletion of the original locus. Both strains were induced with $0.05 \%$ xylose. As can be seen in Figure 4, although they showed a lot of fluctuations, the intensity of the foci was lower in the wild-type strain expressing $\mathrm{SftA}_{(1-105)}$-YFP (from the amyE locus) than in the $\Delta s f t A$ strain expressing the same truncation, suggesting that most of the hexamers present at mid-cell consist of a mixture of the truncated and the intact copies of SftA, which disrupts the function of the hexamer in DNA segregation. To test whether stoichiometry affects the function of the SftA hexamer, different xylose concentrations were used to titrate the truncation in the amyE::sftA $A_{(1-105)}-y f p$ strain. The results are summarized in Table 1 and Figure 5. 


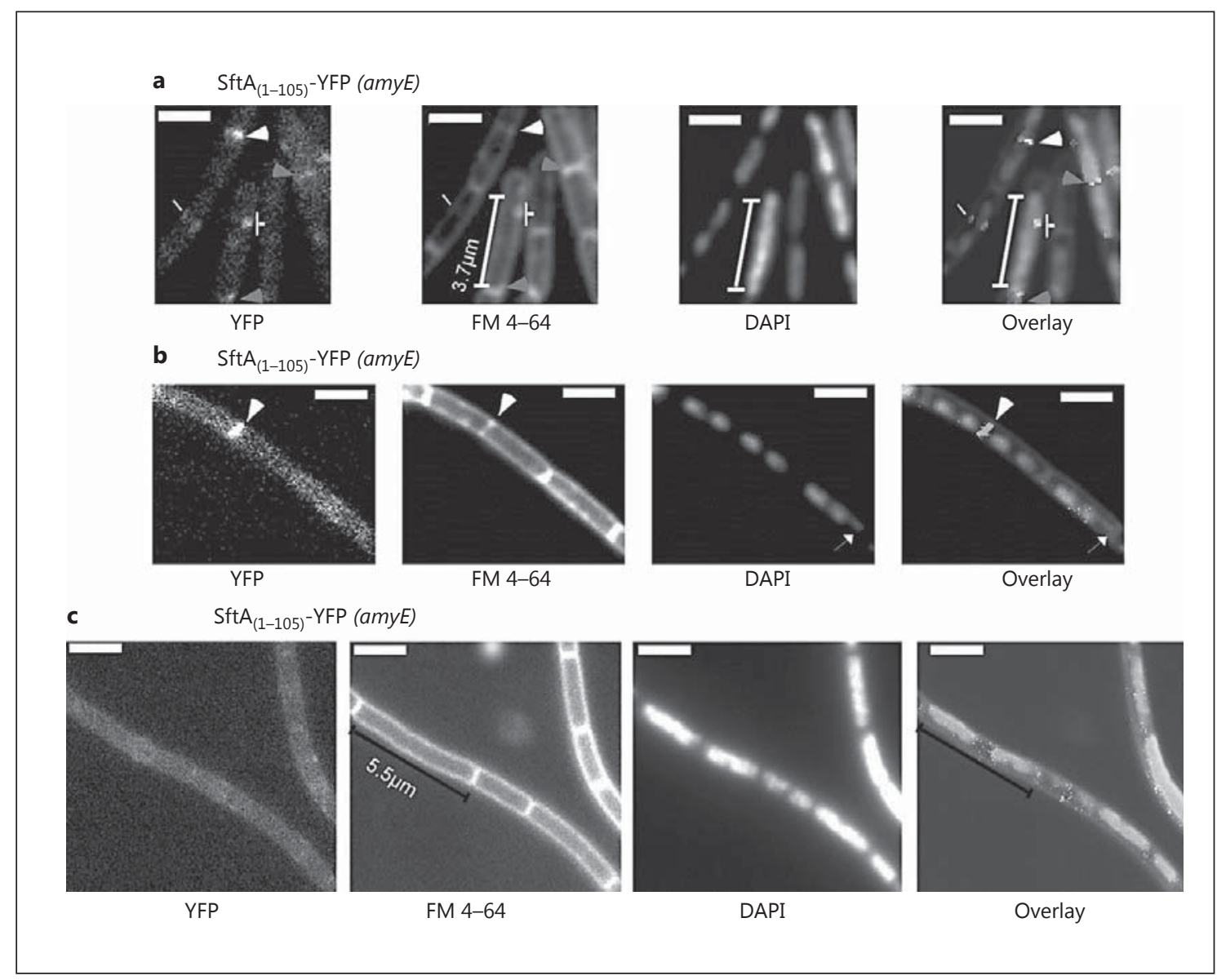

Fig. 5. Localization of $\mathrm{Sft}_{(1-105)}$-YFP cloned at the amyE locus under different induction levels (xylose): $0.02 \%$ xylose (a), $0.05 \%$ xylose (b), and no induction (c). White dashes point to the mid-cell, white arrowheads to new cell poles, grey arrowheads to old cell poles, the small white arrows to a nucleoid bisected by a septum, and the perpendicular lines to SftA localizing at 1 side of the membrane. The elongated cell in a indicated by a ruler has an unsegregated and elongated nucleoid. Scale bars, $2 \mu \mathrm{m}$.

Western blot analysis was performed with anti-GFP antibodies to detect the expression level of the truncation. In comparison, a strain expressing SftA-YFP from its native promoter was used as a control (Fig. 6). Even at a very low induction level ( $0.02 \%$ xylose), the phenotype of the amyE::sftA $A_{(1-105)^{-} y f p}$ was still similar to that of a deletion (Fig. 5a), although the expression level of the truncation was considerably lower than that of the protein under control of the native promoter ( $22 \%$ as calculated by the band intensity in the Western blot and shown in Fig. 6). This could be the outcome of 1 of 2 underlying causes: either the slightest disruption of the stoichiometry of the active hexamer leads to a loss of function, or the short truncations are more favorably incorporated into the hexamer than full-length SftA, ultimately disrupting the function. We consider the prior explanation to be more likely because we could observe a milder $\Delta s f t A$-like segregation defect (in $4 \%$ of the cells vs. $6-7 \%$ in an sftA deletion; Table 1) when no inducer was present (Fig. 5c), i.e., under extremely low expression of the truncation. Note that single $\mathrm{SftA}_{(1-105)}$-YFP molecules would not be visible at the division septum using epifluorescence microscopy (Fig. 5c).

Conversely, a study using linked monomers of $E$. coli FtsK showed that 2 inactive subunits (Walker A or B mutants) within a hexamer did not impair the translocation speed, suggesting that several motor subunits must interact with DNA at any time [Crozat et al., 2010]. Another mechanism has been proposed for dsDNA translocases, including FtsK and SpoIIIE, in which the dsDNA revolves inside a channel formed by the protein, without modifying DNA supercoiling [De-Donatis et al., 2014]. Howev- 


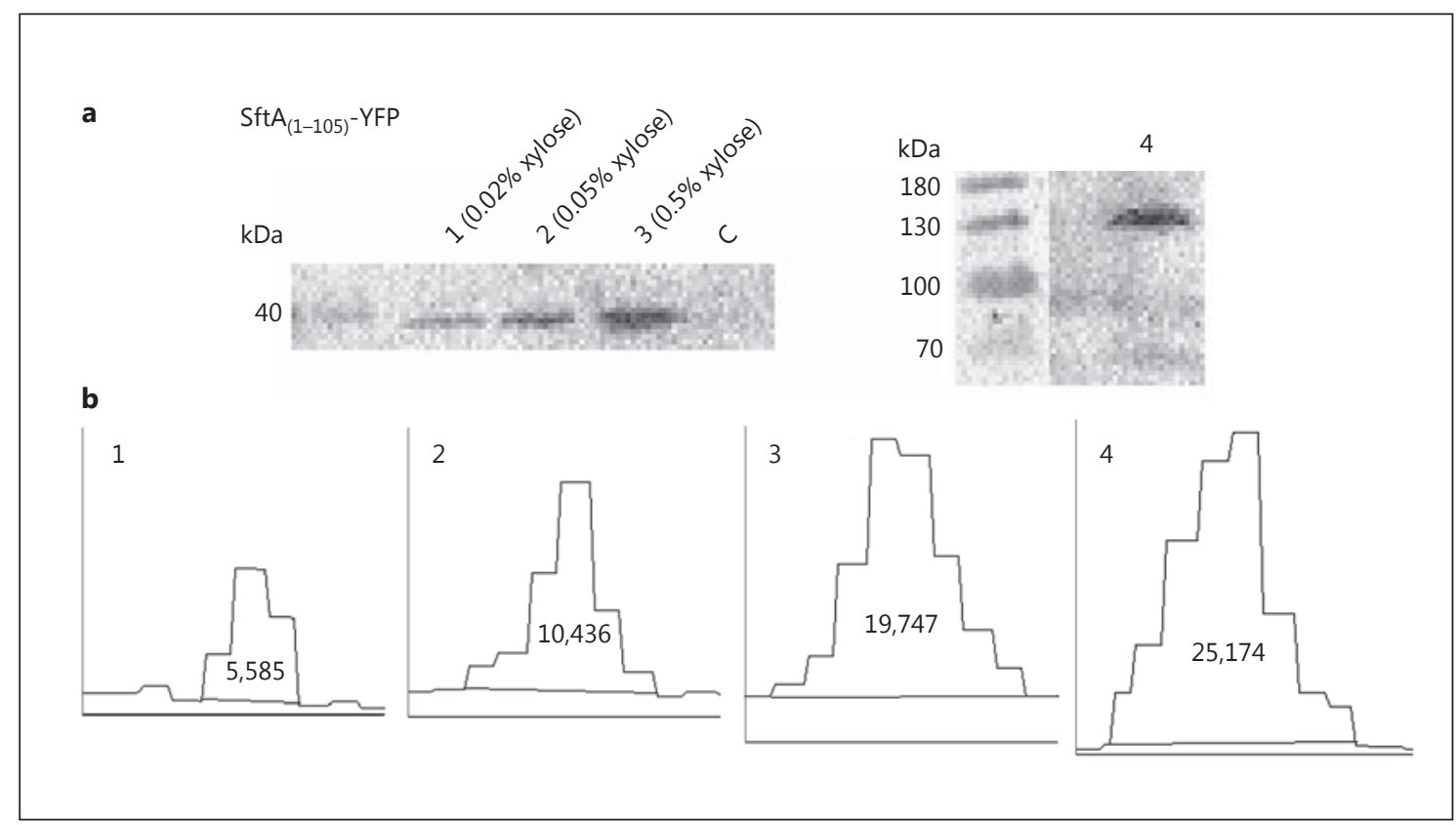

Fig. 6. a Western blot with anti-GFP antibodies for the detection of $\mathrm{Sft}_{(1-105)}$-YFP induced with different xylose concentrations from an ectopic locus. SftA-YFP expressed from the original locus was used as a control (lane 4). b Intensities of the Western blot bands in a in arbitrary units as calculated by ImageJ. Lane $\mathrm{C}=$ no addition of xylose (control).

er, the mechanism implies a loss of activity when 1 subunit is mutated in the ring [Schwarz et al., 2013], which appears in contradiction with the translocation activity retained by a hexamer of FtsK containing 2 opposed inactive subunits [Crozat et al., 2010].

\section{Interaction of SftA with Short dsDNA Fragments}

The capacity of SftA to interact with DNA was tested by gel shift experiments [Kaimer et al., 2009] and surface plasmon resonance. FtsK and SpoIIIE bind to DNA in a nonspecific manner, and show high affinity to specific short polar sequences (KOPS) to confer directionality during DNA translocation [Bigot et al., 2005].

Gel shift experiments were performed in order to determine the formation of a stable protein-DNA complex between purified SftA and short DNA fragments. Increasing concentrations of SftA were incubated with 4 pmol of a 60-bp double-stranded oligonucleotide, either containing 3 copies of the conserved KOPS sequence ( $5^{\prime}$-GGGNAGGG-3'), or containing no KOPS. The formation of a slowly migrating protein DNA complex was observed, and was dependent on the concentration of SftA (Fig. 7a). A clear binding preference towards the KOPS-containing fragment could not be detected in this experiment, but additional experiments are required to obtain definitive evidence for any kind of sequence specificity of SftA. Importantly, SftA showed cooperativity in DNA binding: a 2-fold increase in the concentration of SftA, from 12 to $24 \mathrm{pmol}$, resulted in a drastic increase in SftA/DNA complexes (best seen by the strong reduction in the intensity of the nonbound DNA band), while further increases in protein concentration did not yield a similar binding enhancement (Fig. 7a).

In vitro studies with FtsK showed that, in isolation, the $\gamma$-domain preferentially binds to the KOPS over random DNA [Nolivos et al., 2012; Sivanathan et al., 2006]. SpoIIIE was also shown to prefer SRS-containing DNA to random DNA by a factor of nearly 3 . This was measured using 30-bp-long fragments either containing 2 KOPS sequences or none [Besprozvannaya et al., 2013]. However, in a conflicting study [Cattoni et al., 2014] that investigated the mechanism of DNA binding by SpoIIIE using a combination of single-molecule, biochemical and structural methods, it was shown that SpoIIIE is able to associate to nonspecific DNA with high affinity but no specificity. The DNA binding was unaffected by ATP binding or hydrolysis. Through AFM imaging, SpoIIIE was shown to bind dsDNA as preassembled hexamers without requiring a free DNA end. This is more similar to our findings for SftA, and could mean that the translocase is prob- 
a

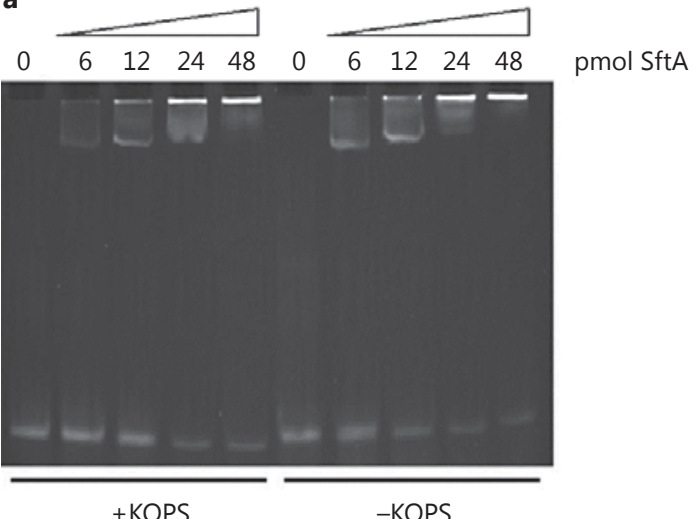

c

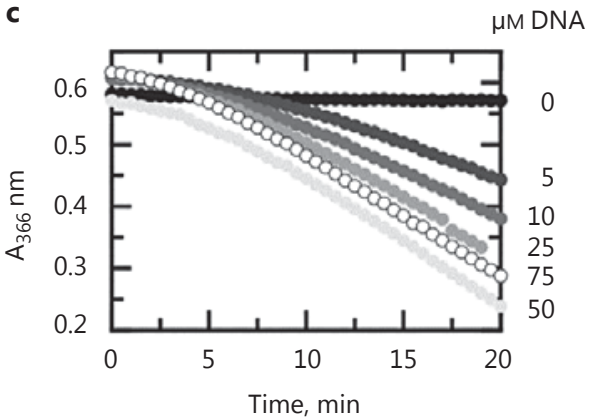

b

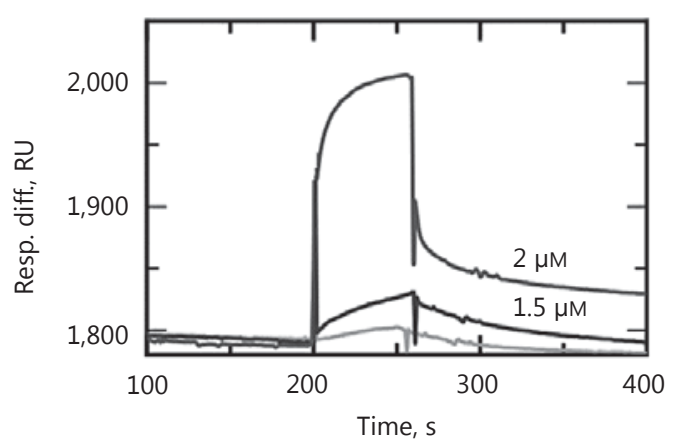

d

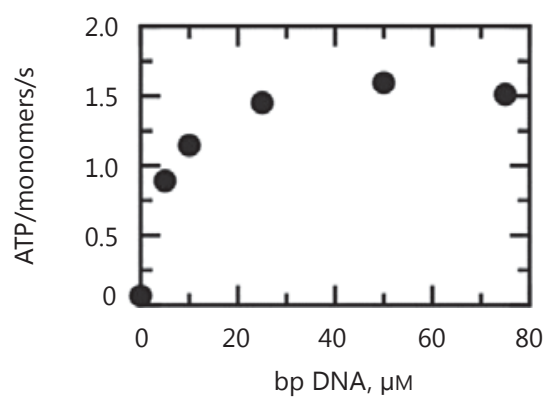

Fig. 7. a Electromobility shift assay. Increasing concentrations of $\mathrm{SftA}$ were incubated with a 60 -bp dsDNA fragment, either containing 3 KOPS sequences (+KOPS) or not (-KOPS). b Surface plasmon resonance experiments. A sensor chip was coated with a 1,500-bp DNA fragment via biotin-streptavidin coupling to a response of 1,800 resonance units (RU). Purified SftA was injected at $200 \mathrm{~s}$ at a concentration of $1 \mu \mathrm{M}$ (light grey), $1.5 \mu \mathrm{M}$ (black) or 2 $\mu \mathrm{M}$ (dark grey). After a 1-min perfusion, protein injection was

ably loaded onto the chromosome anywhere without sequence specificity.

For further characterization of the SftA-DNA interaction, surface plasmon resonance was performed in order to follow the binding of purified SftA to DNA immobilized on a sensor chip. A 1,500-bp PCR fragment biotinylated at the $5^{\prime}$ end was coupled to the sensor chip via a biotin-streptavidin interaction. Injection of $\mathrm{Sft} A$ at different concentrations led to a clear association curve with larger responses at higher protein concentrations (Fig. 7b). Upon washing, dissociation was observed, demonstrating a reversible binding-unbinding equilibrium. Interestingly, the response increased in a nonlinear fashion with the concentration of SftA, whereby an increase from 1.5 to

Localization and Segregation of DNA

Translocase SftA from B. subtilis stopped and the sensor chip was washed with buffer. The graph shows the response difference (Resp. diff.) versus time. c, d Determination of the ATPase activity in a coupled enzyme assay. c $0.06 \mu \mathrm{M}$ of purified SftA were incubated with different concentrations of a 60 -bp dsDNA fragment (as indicated) at $25^{\circ} \mathrm{C}$. ATP hydrolysis is coupled to the oxidation of NADH, reflected by a decrease in $A_{366} \mathrm{~nm}$. d The calculated rate of ATP hydrolysis plotted against the concentration of the DNA substrate.

$2 \mu \mathrm{M}$ resulted in a much higher response than from 1 to $1.5 \mu \mathrm{M}$ (Fig. 7b). This confirms the results obtained in the gel shift assay that SftA binds DNA in a cooperative manner, either during hexamerization, or due to contact between hexamers. Similarly, SpoIIIE was shown to bind DNA as preassembled hexamers [Cattoni et al., 2013].

\section{ATPase Activity Assay}

We have previously shown that SftA can bind to DNA without any sequence specificity, that a hexamer is needed for successful binding since the purified C-terminal failed to achieve detectable binding in gel shift assays, and that binding to circular plasmid DNA induces an ATPase activity that is comparable to SpoIIIE and FtsK [Kaimer 
Table 2. Strains and plasmids used in this study

\begin{tabular}{|c|c|c|c|}
\hline & Relevant genotype & Resistance & Source/reference \\
\hline \multicolumn{4}{|l|}{ E. coli strains } \\
\hline XL 1-Blue & $\begin{array}{l}\text { endA1 gyrA96 }\left(\text { nal }^{\mathrm{R}}\right) \text { thi-1 recA1 relA1 lac } \\
\text { glnV44 } \mathrm{F}^{\prime}\left[:: \operatorname{Tn} 10 \text { proAB }^{+} \operatorname{lacI}^{\mathrm{q}} \Delta(\mathrm{lacZ}) \mathrm{M} 15\right] \\
\text { hsdR17 }\left(\mathrm{r}_{\mathrm{K}}{ }^{-} \mathrm{m}_{\mathrm{K}}{ }^{+}\right)\end{array}$ & Tet & Stratagene \\
\hline BL21 Star (DE3) & $\begin{array}{l}\mathrm{BF}^{-} \text {ompT gal dcm lon hsd } \mathrm{B}_{\mathrm{B}}\left(\mathrm{r}_{\mathrm{B}}{ }^{-} \mathrm{m}_{\mathrm{B}}{ }^{-}\right) \lambda(\mathrm{DE} 3 \\
{[\mathrm{lacI} \text { lacUV5-T7 gene } 1 \text { ind1 sam7 nin5]) }} \\
{\left[\mathrm{malB}^{+}\right]_{\mathrm{K}-12}\left(\lambda^{S}\right)}\end{array}$ & none & Invitrogen \\
\hline \multicolumn{4}{|l|}{ Plasmids } \\
\hline pSG1729 & & & Lewis and Marston [1999] \\
\hline pSG1193y & & & Feucht and Lewis [2001] \\
\hline pSG1164y & & & Kidane et al. [2004] \\
\hline pETDUET-1 & & & Novagen \\
\hline \multicolumn{4}{|l|}{ B. subtilis strains } \\
\hline PY79 & wild-type & none & Youngman et al. [1983] \\
\hline NEJ1 & amyE::sft $A_{(1-490)}-y f p$ & Spec & this study \\
\hline NEJ2 & $s f t A_{(1-246)}-y f p$ & $\mathrm{Cm}$ & this study \\
\hline NEJ3 & $s f t A_{(1-137)-y f p}$ & $\mathrm{Cm}$ & this study \\
\hline NEJ4 & $s f t A_{(1-105)}-y f p$ & $\mathrm{Cm}$ & this study \\
\hline NEJ5 & $s f t A_{(1-67)^{-}} y f p$ & $\mathrm{Cm}$ & this study \\
\hline NEJ6 & $s f t A_{(1-50)}-y f p$ & $\mathrm{Cm}$ & this study \\
\hline NEJ7 & $s f t A_{(1-34)^{-}} y f p$ & $\mathrm{Cm}$ & this study \\
\hline CK150 & sftA::tet & Tet & Kaimer et al. [2009] \\
\hline NEJ8 & amyE::sftA ${ }_{(1-105)}-y f p$ sftA::tet & Spec/Tet & this study \\
\hline NEJ9 & amyE::sftA $(1-67)^{-y f p}$ sftA::tet & Spec/Tet & this study \\
\hline NEJ10 & amyE::sft $A_{(21-439)^{-} y f p}$ & Spec & this study \\
\hline NEJ11 & $a m y E:: s f t A_{(21-100)}-y f p$ & Spec & this study \\
\hline NEJ12 & amyE::sftA $(21-100)^{-y f p}$ sftA::tet & Spec/Tet & this study \\
\hline NEJ13 & $a m y E:: s f t A_{(21-67)}-y f p$ & Spec & this study \\
\hline NEJ14 & amyE::sftA ${ }_{(21-67)}-y f p$ sftA::tet & Spec/Tet & this study \\
\hline PG2664 & $p f k A-y f p$ & $\mathrm{Cm}$ & Graumann laboratory stock \\
\hline CK55 & spoIIIE-yfp & $\mathrm{Cm}$ & Kaimer et al. [2009] \\
\hline NEJ15 & $a m y E:: s f t A_{(61-439)}-y f p$ & Spec & this study \\
\hline CK70 & $s f t A-y f p$ & $\mathrm{Cm}$ & Kaimer et al. [2009] \\
\hline
\end{tabular}

et al., 2009]. We next tested if 60-bp fragments are sufficient to induce ATPase activity in a coupled enzyme assay. A clear increase of the ATP hydrolysis rate was observed in the presence of increasing DNA concentrations (Fig. 7c). However, only a maximal rate of approximately $1.5 \mathrm{ATP} /$ monomer/s was measured (Fig. 7d). Kaimer et al. [2009] observed a 6.5-fold higher ATPase activity when supercoiled plasmid DNA was used, with the same molecular amount of base pairs.

This reduced activity is possibly explained by the functional mechanism of DNA translocases: if SftA indeed moves quickly along DNA in an ATP-dependent manner, as is claimed for FtsK [Bigot et al., 2005] and SpoIIIE [Besprozvannaya et al., 2013], the protein would rapidly reach the end of a short fragment and dissociate. ATP hy- drolysis would drop to a very low rate until reassociation with DNA, which again induces increased ATPase activity. When short linear DNA fragments are present, the overall ATP hydrolysis rate would therefore be reduced due to permanent dissociation and reassociation, which is avoided when longer or circular DNA is available as a substrate. Interestingly, we observed a similar maximal ATP hydrolysis rate of $1.7 \mathrm{ATP} / \mathrm{monomer} / \mathrm{s}$ for the isolated C-terminal domain SftA-C with circular plasmid DNA as the substrate [Kaimer et al., 2009]. It was reasoned that the C-terminal domain, which is not able to oligomerize, cannot establish a stable interaction with DNA and therefore also rapidly dissociates. Therefore, in both cases, measurements probably reflect a reduced ATPase activity because the protein-DNA interaction re- 
mains transient. However, Besprozvannaya et al. [2013] showed that SpoIIIE exhibits length-dependent ATPase activity, in which case 61-bp DNA fragments were determined as the minimal dsDNA fragment length that stimulates maximal ATPase activity. This seems not to be the case for SftA, where longer fragments are indeed required.

\section{Conclusions}

Our data show that the short region from amino acid 20 to 67 is sufficient for targeting SftA to the division site, suggesting that $\mathrm{SftA}$ is a bona fide component of the division machinery that is recruited by a protein-protein interaction. The deletion of 20 amino acids from the $\mathrm{N}$ terminus did not abrogate recruitment to mid-cell, indicating that SftA is likely not a membrane-integral protein, but truly cytosolic when not present at the division septum. This was confirmed by cell fractionation experiments, showing that $\mathrm{SftA}$ is a largely soluble protein, with a minor fraction being associated with the membrane, most likely through the interaction with a truly membrane-associated division protein.

Moreover, we showed that SftA binds cooperatively to DNA, which is reflected by a nonlinear increase in DNA binding with increasing protein concentrations. This is in agreement with the finding that $\mathrm{SftA}$ forms stable hexamers, so a certain threshold of its concentration will enable stable DNA binding within the hexameric assembly. In a previous study, we showed that SftA has a high ATPase activity that is comparable to SpoIIIE and FtsK when a circular plasmid was used as a substrate [Kaimer et al., 2009]. However, with short DNA fragments of just $60 \mathrm{bp}$, ATPase activity is considerably lower, suggesting that SftA performs large processive translocation events and rapidly dissociates from short DNA molecules.

Our experiments show that, despite the fact that SftA bears many similarities to SpoIIIE and FtsK in terms of sequence and function, some distinct features may be important for its role as a DNA pump prior to septum closure. Unlike FtsK and SpoIIIE, SftA is soluble and is recruited to the septum via another component of the divisome. While the $\mathrm{N}$-terminal regions of SpoIIIE and FtsK each contain 4 transmembrane helices that incorporate them into the membrane [Liu et al., 1998; Yu et al., 1998], only a short span within the $\mathrm{N}$-terminus of $\mathrm{SftA}$ is required to target it to the division septum. Since the deletion of SftA and that of a Walker A mutant have the same phenotype [Kaimer et al., 2009], we suggest that the Nterminal part of SftA has no intrinsic function other than

Localization and Segregation of DNA

Translocase SftA from B. subtilis mid-cell targeting. This is in contrast to E. coli FtsK, in which a mutation within a loop in the $\mathrm{N}$-terminus resulted in uncoupling invagination of the inner and outer membranes [Berezuk et al., 2014].

$\mathrm{SftA}$ forms hexamers in vitro. Interestingly, our localization experiments show that the function of the protein is lost when the hexamer is disrupted by the incorporation of a truncated version of the protein, suggesting that to transport DNA, the C-terminal domains perform (possibly sequential) power strokes that do not permit the omission of 1 of the 6 domains. In contrast to this, SpoIIIE can still pump DNA if the hexamer contains a nonfunctional subunit [Liu et al., 2015].

SftA, like FtsK, is present in most cells in the division septa, and is recruited as an early component of the divisome [Kaimer et al., 2009]. SpoIIIE, on the other hand, is not generally recruited to the division machinery, but assembles at sites where DNA becomes trapped within the septal membranes. Additionally, SpoIIIE plays a major role during sporulation, whereby it promotes chromosome translocation to the forespore in addition to membrane fission [Shin et al., 2015].

$\mathrm{SftA}$ assists in the resolution of chromosome dimers, but is not required to activate the recombinases, and its deletion leads only to a mild segregation defect [Kaimer et al., 2011]. Thus, FtsK plays multiple roles in linking division to segregation and in actively contributing to the segregation of the terminus region of chromosomes, in addition to active involvement in the resolution of dimer formation [Stouf et al., 2013]. These are functions that have been distributed to several different translocases and division proteins in B. subtilis.

\section{Materials and Methods}

\section{Growth Conditions}

The bacterial strains and plasmids used in this study are listed in Table 2, and the oligonucleotides are detailed in online supplementary Table S1. E. coli strain XL1-Blue (Stratagene) was used for the construction and propagation of plasmids and E. coli strain BL21 Star DE3 (Invitrogen) for the heterologous overexpression of proteins. All B. subtilis strains were derived from the prototrophic wild-type strain PY79. Cells were grown in Luria-Bertani-rich medium at 37 or $30^{\circ} \mathrm{C}$, or in minimal medium containing S7 50 salts [Jaacks et al., 1989] at $25^{\circ} \mathrm{C}$. Media were supplemented with antibiotics where appropriate (ampicillin $100 \mathrm{mg} \mathrm{mL}^{-1}$, chloramphenicol $5 \mathrm{mg} \mathrm{mL}^{-1}$, spectinomycin $100 \mathrm{mg} \mathrm{mL}^{-1}$, kanamycin $10 \mathrm{mg}$ $\mathrm{mL}^{-1}$, tetracycline $10 \mathrm{mg} \mathrm{mL}^{-1}$ ).

\section{Strain Construction}

Strain amyE::sftA $A_{(1-439)}-y f p$ was obtained by cloning the N-terminal region of SftA into the ApaI and EcoRI sites of pSG1193y [Lewis and Marston, 1999], which integrates at the amylase locus 
by a double-crossover event. Strains bearing fluorescent truncation variants of SftA $\left[s f t A_{(1-246)^{-}} y f p, s f t A_{(1-137)^{-}} y f p, s f t A_{(1-105)^{-}} y f p\right.$, $s f t A_{(1-67)^{-}} y f p, s f t A_{(1-34)^{-}} y f p$, and $\left.s f t A_{(1-50)^{-}} y f p\right]$ were constructed for each construct by cloning a corresponding 500-bp fragment of the SftA N-terminal into the ApaI and EcoRI sites of pSG1164y [Kidane et al., 2004] and by transformation of PY79 with the resulting vector which integrates at the original locus of the gene by a single-crossover event. Each of the created strains expresses a part of the N-terminal region of SftA fused to a YFP from the native promoter. For the truncation copies that are shorter than 500 $\mathrm{bp}$, a region upstream of the coding sequence was included in the construct in a way that ensured the total length of the cloned region was always around $500 \mathrm{bp}$. A full copy of the gene was still present and expressed from the xylose promoter of the vector.

To obtain an $s f t A:: t e t$ deletion in a truncation background [amyE::sftA $A_{(1-105)^{-}-y f p} s f t A:: t e t$ and amyE::sftA $A_{(1-67)^{-}} y f p$ sftA::tet], the SftA fragments were first cloned each into the ApaI and EcoRI sites of pSG1193y. PY79 was transformed with the vectors. Chromosomal DNA of CK150 [Kaimer et al., 2009] was used for transformation of PY79 competent cells of the generated truncationcontaining strains. Deletion was confirmed by test PCR, and strains were viewed through a microscope in the exponential phase after induction with $0.1 \%$ xylose. Constructs amyE::sftAN ${ }_{(21-439)}$ $y f p$ and $a m y E:: s f t A N_{(61-439)}-y f p$ were created by cloning the N-terminal fragments of SftA which are missing the first 20 and the first 60 amino acids, respectively, between the ApaI and the EcoRI sites of pSG1193y. The plasmids were transformed into cells of competent sftA::tet strain.

\section{Cell Fractionation Experiments}

Cell fractionation experiments were performed according to the protocol by Zweers et al. [2009]. Briefly, cells were grown overnight in Luria-Bertani medium, collected by centrifugation, and resuspended in protoplast buffer $(100 \mathrm{mM}$ Tris- $\mathrm{HCl}, \mathrm{pH} 8.2,20 \mathrm{mM}$ $\mathrm{MgCl}_{2}, 20 \%$ sucrose, $1 \mathrm{mg} / \mathrm{mL}$ lysozyme, $0.01 \% \mathrm{DNase}$, and complete protease inhibitors). After a 30 -min incubation at $37^{\circ} \mathrm{C}$, the cell wall fraction was separated from the protoplasts by centrifugation $\left(10 \mathrm{~min}, 4,000 \mathrm{~g}, 4^{\circ} \mathrm{C}\right)$. Protoplasts were resuspended in disruption buffer (50 mM Tris-HCl, pH 8.2, 2.5 mM EDTA) and disrupted with a French press. Cellular debris were removed by centrifugation (10 $\mathrm{min}, 4,000 \mathrm{~g}, 4^{\circ} \mathrm{C}$ ), and the supernatant was ultracentrifuged (30 min, 200,000 $\mathrm{g}, 4^{\circ} \mathrm{C}$ ). Next, the supernatant fraction with the cytosolic proteins was collected. The pellet was resuspended in solubilization buffer (20 mM Tris, $\mathrm{pH}$ 8.0, 10\% glycerol, $50 \mathrm{mM} \mathrm{NaCl}$, $0.03 \%$ DDM [n-dodecyl- $\beta$-maltoside]) and incubated overnight at $4^{\circ} \mathrm{C}$. Solubilized membrane proteins were collected by centrifugation in the supernatant $\left(15 \mathrm{~min}, 100,000 \mathrm{~g}, 4^{\circ} \mathrm{C}\right)$. The fractions thus obtained were analyzed by SDS-PAGE and Western blotting.

\section{Fluorescence Microscopy}

For fluorescence microscopy, B. subtilis cells were grown in $\mathrm{S}_{50}$ minimal medium at $25^{\circ} \mathrm{C}$ under shaking conditions until exponential growth. Three microliters of cells were transferred on an agarose slide - a glass slide (microscope slides standard, Roth) coated with an agarose layer (S750 minimal medium, $10 \mathrm{mg} / \mathrm{mL}$ agarose) - and covered with a cover slip (Roth). Conventional light microscopy was performed using a Zeiss Observer Z1 (Carl Zeiss) with an oil immersion objective $(100 \times$ magnification, 1.45 numerical aperture, alpha Plan-FLUAR, Carl Zeiss) and a CCD camera (CoolSNAP EZ, Photometrics), or with a BX51 microscope (Olympus) with a Cool Snap
EZ camera (Photometrics) and a xenon light source (Olympus). The cells were treated with red fluorescent membrane stain FM 4-64 (excitation: $515 \mathrm{~nm} /$ emission: $640 \mathrm{~nm}$, final concentration $1 \mathrm{nM}$ ) and DNA intercalating blue fluorescent dye DAPI (excitation: 358 $\mathrm{nm} / \mathrm{emission}$ : $461 \mathrm{~nm}$, final concentration $0.72 \mathrm{nM}$ ) and incubated for $2 \mathrm{~min}$ at room temperature prior to microscopy. Electronic data were processed using Metamorph 7.5.5.0 software (Molecular Devices, Sunnyvale, CA, USA), which also allows the calibration of the fluorescence intensity and pixel size to determine the cell length.

\section{Corrected Fluorescence Intensity Measurement}

The strains $\left[s f t A_{(1-105)^{-}} y f p, s f t A_{(1-67)}-y f p\right.$, and $\left.s f t A-y f p\right]$ were first grown in parallel in $\mathrm{S} 750$ minimal medium at $25^{\circ} \mathrm{C}$ until an $\mathrm{OD}_{600}$ of around 0.7 was reached. Three microliters of each culture were then added on an agarose slide and the strains were subsequently viewed through a microscope on a Zeiss Observer Z1 with an exposure time of $1 \mathrm{~s}$. Foci fluorescence intensity measurement was done using the Java-based image processing program ImageJ (Wayne Rasband, National Institutes of Health), and the numbers obtained were in arbitrary units. The mean background fluorescence was determined from 100 puncta from the wild-type strain, and was subtracted from the measured intensity of each focus to correct for the basic fluorescence level of B. subtilis under the YFP channel.

\section{Calculation of Western Blot Band Intensity}

$\mathrm{Sft}_{(1-105)}$-YFP was induced with different levels of xylose to evaluate the effect of the incorporation of truncated copies of the protein on the function of the hexamers. The expression level of the construct was assessed on the Western blots. Briefly, the intensities of bands were compared according to their grayscale (http:// www.lukemiller.org/journal/2007/08/quantifying-western-blotswithout.html).

\section{Electrophoretic Mobility Shift Assay}

Pairs of complementary oligonucleotides were annealed to form double-stranded fragments (see online suppl. Table S1). Oligonucleotides were mixed in equal amounts $(10 \mu \mathrm{M})$ in a buffer containing $10 \mathrm{~mm}$ Tris- $\mathrm{HCl}, \mathrm{pH}$ 8.0, $50 \mathrm{~mm} \mathrm{NaCl}$. After complete denaturation at $95^{\circ} \mathrm{C}$ for $5 \mathrm{~min}$, the samples were slowly cooled to room temperature to promote annealing. The formation of double-stranded fragments was confirmed by agarose gel electrophoresis. Annealing of oligonucleotides 1250 and 1251 resulted in the -KOPS fragment, the +KOPS fragment consisted of oligonucleotides 1882 and 1883 . The DNA fragments were incubated with increasing amounts of SftA according to the protocol described in Kaimer et al. [2009] and analyzed on a native gradient polyacrylamide gel (5-15\%).

\section{Surface Plasmon Resonance}

Measurements were carried out using a Biacore 3000 system (GE Healthcare) and streptavidin-coated Biacore SA sensor chips. After conditioning the sensor chip with a washing solution (1 M $\mathrm{NaCl}, 0.05 \mathrm{M} \mathrm{NaOH}$ ), biotinylated DNA fragments (1,500 bp) generated by PCR with primers 1162 and 1164 (online suppl. Table S1) were immobilized on 1 channel of the sensor chip according to the manufacturer's instructions, resulting in a response of 1,800 resonance units. The signal of a blank channel served as reference and was subtracted in all measurements. Purified SftA was injected at $200 \mathrm{~s}$ at various concentrations at a continuous flow rate of 
$20 \mu \mathrm{L} \mathrm{min}{ }^{-1}$ in $0.1 \mathrm{M}$ Tris- $\mathrm{HCl}, \mathrm{pH} 8.0,0.1 \mathrm{M} \mathrm{NaCl}$. All buffers were filtered and degassed before use. After a 1-min perfusion, protein injection was stopped at $260 \mathrm{~s}$ and the sensor chip was washed with buffer.

\section{ATPase Activity Assay}

The ATPase activity of purified SftA was determined as prescribed in Bath et al. [2000]. Briefly, the enzyme assay couples 2 reactions: the conversion of phosphoenolpyruvate to pyruvate, and the conversion of pyruvate to lactate, catalyzed by pyruvate kinase and lactate dehydrogenase, respectively. The latter reaction results in the oxidation of NADH to $\mathrm{NAD}^{+}$, which can be followed by the decrease in absorbance measured at $366 \mathrm{~nm}$. The phosphate resulting from the pyruvate kinase reaction is used to regenerate
ADP, the product of ATP hydrolysis by SftA. In summary, $1 \mathrm{~mol}$ of NADH is converted for $1 \mathrm{~mol}$ of ATP. The 60-bp DNA substrate was obtained by annealing oligonucleotides 1882 and 1883 (see online suppl. Table S1).

\section{Acknowledgements}

This work was supported by the Katholischer Akademischer Ausländer-Dienst (KAAD), by the BMBF-funded graduate training group NANOKAT, by the LOEWE-funded Centre for Synthetic Microbiology (SYNMIKRO) at the Philipps-Universität Marburg, and by the Deutsche Forschungsgemeinschaft (DFG).

\section{References}

Aussel L, Barre F-X, Aroyo M, Stasiak A, Stasiak $\mathrm{AZ}$, Sherratt D: FtsK is a DNA motor protein that activates chromosome dimer resolution by switching the catalytic state of the XerC and XerD recombinases. Cell 2002;108:195205.

Barre FX: FtsK and SpoIIIE: the tale of the conserved tails. Mol Microbiol 2007;66:10511055.

Barre FX, Sherratt D: Chromosome dimer resolution; in Higgins NP (ed): The Bacterial Chromosome. Washington, ASM, 2005, pp 513524.

Bath J, Wu LJ, Errington J, Wang JC: Role of $B a$ cillus subtilis SpoIIIE in DNA transport across the mother cell-prespore division septum. Science 2000;290:995-997.

-Berezuk AM, Goodyear M, Khursigara CM: Sitedirected fluorescence labeling reveals a revised n-terminal membrane topology and functional periplasmic residues in the Escherichia coli cell division protein FtsK. J Biol Chem 2014;289:23287-23301.

-Besprozvannaya M, Pivorunas VL, Feldman Z, Burton BM: SpoIIIE protein achieves directional DNA translocation through allosteric regulation of ATPase activity by an accessory domain. J Biol Chem 2013;288:28962-28974.

Bigot S, Saleh OA, Lesterlin C, Pages C, El Karou M, Dennis C, Grigoriev M, Allemand JF, Barre FX, Cornet F: KOPS: DNA motifs that control E. coli chromosome segregation by orienting the FtsK translocase. EMBO J 2005; 24:3770-3780.

Bigot S, Sivanathan V, Possoz C, Barre FX, Cornet F: FtsK, a literate chromosome segregation machine. Mol Microbiol 2007;64:1434-1441.

-Biller SJ, Burkholder WF: The Bacillus subtilis SftA (YtpS) and SpoIIIE DNA translocases play distinct roles in growing cells to ensure faithful chromosome partitioning. $\mathrm{Mol} \mathrm{Mi}$ crobiol 2009;74:790-809.
Bobay LM, Rocha EP, Touchon M: The adaptation of temperate bacteriophages to their host genomes. Mol Biol Evol 2013;30:737751.

Burton B, Dubnau D: Membrane-associated DNA transport machines. Cold Spring Harb Perspect Biol 2010;2:a000406.

Cattoni DI, Chara O, Godefroy C, Margeat E, Trigueros S, Milhiet PE, Nollmann M: SpoIIIE mechanism of directional translocation involves target search coupled to sequence-dependent motor stimulation. EMBO Rep 2013 14:473-479.

Cattoni DI, Thakur S, Godefroy C, Le Gall A, LaiKee-Him J, Milhiet PE, Bron P, Nollmann M: Structure and DNA-binding properties of the Bacillus subtilis SpoIIIE DNA translocase revealed by single-molecule and electron microscopies. Nucleic Acids Res 2014;42:26242636.

Collins BE, Ling FY, Duzdevich D, Greene EC: DNA curtains: novel tools for imaging protein-nucleic acid interactions at the singlemolecule level. Methods Cell Biol 2014;123: 217-234.

Crozat E, Meglio A, Allemand JF, Chivers CE, Howarth M, Venien-Bryan C, Grainge I, Sherratt DJ: Separating speed and ability to displace roadblocks during DNA translocation by FtsK. EMBO J 2010;29:1423-1433.

Crozat E, Rousseau P, Fournes F, Cornet F: The FtsK family of DNA translocases finds the ends of circles. J Mol Microbiol Biotechnol 2014;24:396-408

De-Donatis GM, Zhao Z, Wang S, Huang LP, Schwartz C, Tsodikov OV, Zhang H, Haque F, Guo P: Finding of widespread viral and bacterial revolution dsDNA translocation motors distinct from rotation motors by channel chirality and size. Cell Biosci 2014;4:30.

Dubarry N, Barre FX: Fully efficient chromosome dimer resolution in Escherichia coli cells lacking the integral membrane domain of FtsK. EMBO J 2010;29:597-605.
Feucht A, Lewis PJ: Improved plasmid vectors for the production of multiple fluorescent protein fusions in Bacillus subtilis. Gene 2001;264:289-297.

Graham JE, Sivanathan V, Sherratt DJ, Arciszewska LK: FtsK translocation on DNA stops at XerCD-dif. Nucleic Acids Res 2010:38:72-81.

Grainge I, Bregu M, Vazquez M, Sivanathan V, Ip SC, Sherratt DJ: Unlinking chromosome catenanes in vivo by site-specific recombination. EMBO J 2007;26:4228-4238.

Hallet B, Sherratt DJ: Transposition and site-specific recombination: adapting DNA cut-andpaste mechanisms to a variety of genetic rearrangements. FEMS Microbiol Rev 1997;21: 157-178.

Jaacks K, Healy J, Losick R, Grossman A: Identification and characterization of genes controlled by the sporulation-regulatory gene spoOH in Bacillus subtilis. J Bacteriol 1989; 171:4121-4129.

- Kaimer C, Gonzaler-Pastor E, Graumann PL: SpoIIIE and a novel type of DNA translocase, SftA, couple chromosome segregation with cell division in Bacillus subtilis. Mol Microbiol 2009;74:810-825.

Kaimer C, Graumann PL: Players between the worlds: Multifunctional DNA translocases. Curr Opin Microbiol 2011;14:719-725.

Kaimer C, Schenk K, Graumann PL: Two DNA translocases synergistically affect chromosome dimer resolution in Bacillus subtilis. J Bacteriol 2011;193:1334-1340.

Kidane D, Sanchez H, Alonso JC, Graumann PL: Visualization of DNA double-strand break repair in live bacteria reveals dynamic recruitment of Bacillus subtilis RecF, RecO and RecN proteins to distinct sites on the nucleoids. Mol Microbiol 2004;52:1627-1639.

Lee JY, Finkelstein IJ, Arciszewska LK, Sherratt DJ, Greene EC: Single-molecule imaging of FtsK translocation reveals mechanistic features of protein-protein collisions on DNA. Mol Cell 2014;54:832-843.
Localization and Segregation of DNA

Translocase SftA from B. subtilis
J Mol Microbiol Biotechnol 2017;27:29-42 DOI: $10.1159 / 000450725$ 
Lee JY, Finkelstein IJ, Crozat E, Sherratt DJ, Greene EC: Single-molecule imaging of DNA curtains reveals mechanisms of KOPS sequence targeting by the DNA translocase FtsK. Proc Natl Acad Sci USA 2012;109:65316536.

Lewis PJ, Marston AL: GFP vectors for controlled expression and dual labelling of protein $\mathrm{fu}-$ sions in Bacillus subtilis. Gene 1999;227:101109.

Liu G, Draper GC, Donachie WD: FtsK is a bifunctional protein involved in cell division and chromosome localization in Escherichia coli. Mol Microbiol 1998;29:893-903.

Liu N, Chistol G, Bustamante C: Two-subunit DNA escort mechanism and inactive subunit bypass in an ultra-fast ring ATPase. eLife 2015;4:e09224.

Ludwig H, Homuth G, Schmalisch M, Dyka FM, Hecker M, Stülke J: Transcription of glycolytic genes and operons in Bacillus subtilis: evidence for the presence of multiple levels of control of the gapA operon. Mol Microbiol 2001;41:409-422.

-Massey TH, Mercogliano CP, Yates J, Sherratt DJ, Löwe J: Double-stranded DNA translocation: structure and mechanism of hexameric FtsK. Mol Cell 2006;23:457-469.

- May PF, Zawadzki P, Sherratt DJ, Kapanidis AN, Arciszewska LK: Assembly, translocation, and activation of XerCD-dif recombination by FtsK translocase analyzed in real-time by FRET and two-color tethered fluorophore motion. Proc Natl Acad Sci USA 2015;112: E5133-E5141.
Nolivos S, Touzain F, Pages C, Coddeville M, Rousseau P, El Karoui M, Le Bourgeois P, Cornet F: Co-evolution of segregation guide DNA motifs and the FtsK translocase in bacteria: identification of the atypical Lactococcus lactis KOPS motif. Nucleic Acids Res 2012;40: 5535-5545.

Pease PJ, Levy O, Cost GJ, Gore J, Ptacin JL, Sherratt $\mathrm{D}$, Bustamante $\mathrm{C}$, Cozzarelli NR: Sequence-directed DNA translocation by purified FtsK. Science 2005;307:586-590.

Schwarz FW, Toth J, van Aelst K, Cui G, Clausing S, Szczelkun MD, Seidel R: The helicase-like domains of type III restriction enzymes trigger long-range diffusion along DNA. Science 2013;340:353-356.

Sciochetti SA, Piggot PJ, Blakely GW: Identification and characterization of the dif site from Bacillus subtilis. J Bacteriol 2001;183:10581068.

- Sciochetti SA, Piggot PJ, Sherratt DJ, Blakely G: The ripX locus of Bacillus subtilis encodes a site-specific recombinase involved in proper chromosome partitioning. J Bacteriol 1999; 181:6053-6062.

-Sharpe ME, Errington J: Postseptational chromosome partitioning in bacteria. Proc Natl Acad Sci USA 1995;92:8630-8634.

Sherratt DJ, Arciszewska LK, Blakely G, Colloms S, Grant K, Leslie N, McCulloch R: Site-specific recombination and circular chromosome segregation. Philos Trans R Soc Lond B Biol Sci 1995;347:37-42.
Shin JY, Lopez-Garrido J, Lee S-H, Diaz-Celis C, Fleming T, Bustamante C, Pogliano K: Visualization and functional dissection of coaxial paired SpoIIIE channels across the sporulation septum. eLife 2015;4:e06474.

Sivanathan V, Allen MD, de Bekker C, Baker R, Arciszewska LK, Freund SM, Bycroft M, Lowe J, Sherratt DJ: The FtsK $\gamma$ domain directs oriented DNA translocation by interacting with KOPS. Nat Struct Mol Biol 2006;13:965-972.

-Stouf M, Meile JC, Cornet F: FtsK actively segregates sister chromosomes in Escherichia coli. Proc Natl Acad Sci USA 2013;110:1115711162.

Studier FW: Protein production by auto-induction in high-density shaking cultures. Protein Expr Purif 2005;41:207-234.

Yates J, Zhekov I, Baker R, Eklund B, Sherratt DJ, Arciszewska LK: Dissection of a functional interaction between the DNA translocase, FtsK, and the XerD recombinase. Mol Microbiol 2006;59:1754-1766.

-Youngman PJ, Perkins JB, Losick R: Genetic transposition and insertional mutagenesis in Bacillus subtilis with Streptococcus faecalis transposon Tn917. Proc Natl Acad Sci USA 1983;80:2305-2309.

-Yu XC, Tran AH, Sun Q, Margolin W: Localization of cell division protein FtsK to the Escherichia coli septum and identification of a potential n-terminal targeting domain. J Bacteriol 1998; 180:1296-1304.

Zweers JC, Wiegert T, van Dijl JM: Stress-responsive systems set specific limits to the overproduction of membrane proteins in Bacillus subtilis. Appl Environ Microbiol 2009;75: 7356-7364. 